\title{
La prise en charge odonto-stomatologique des patients infectés par le VIH : réalités, entraves et solutions
}

\author{
Boukais H, Zerrouki W, Sahraoui M, Terkmani M, Mokrani M, Zeraoui O \\ Service de Pathologie et Chirurgie buccale, CHU Béni-Messous, Alger, Algérie \\ boukais.hamid@caramail.com
}

On définit le SIDA comme l'ensemble des manifestations cliniques du déficit de l'immunité cellulaire due à l'atteinte des lymphocytes T4; le SIDA représente la forme majeure de l'infection par le VIH.

Les signes buccaux de la maladie constituent parfois des prodromes, leur découverte permet alors de suspecter la maladie et de mettre en œuvre les examens nécessaires pour sa confirmation. Ainsi, le chirurgien dentiste est pleinement impliqué dans la prise en charge de ces patients, par la reconnaissance et le traitement des lésions buccales qu'elle engendre, et la remise en état de la cavité buccale.

Pour connaître l'attitude des chirurgiens dentistes face à un malade atteint de SIDA consultant au cabinet dentaire, nous avons mené une enquête en Algérie sur un échantillon représentatif de 500 chirurgiens dentistes tous secteurs et toutes spécialités confondus.

Les résultats de cette enquête mettent en évidence un déficit de prise en charge de ces malades en odontologie en raison d'une surestimation du risque de contamination, d'une méconnaissance des risques et de leur prévention, et par manque d'information.

Cette réalité et la nette augmentation du nombre de cas dans notre pays et dans le monde, imposent de mettre en œuvre des actions sur le terrain.

A travers cette communication orale, nous vous rapporterons les résultats de l'enquête, nous rappellerons brièvement les signes cliniques bucco-dentaires en rapport avec cette infection et nous proposerons une stratégie en démystifiant la prise en charge d'un patient infecté par le VIH. 